\title{
miR-34a inhibits the migration and invasion of esophageal squamous cell carcinoma by targeting Yin Yang-1
}

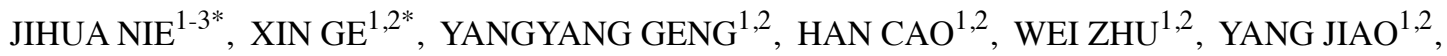 \\ JINCHANG WU ${ }^{4}$, JUNDONG ZHOU ${ }^{4}$ and JIANPING CAO ${ }^{1,2}$
}

\begin{abstract}
${ }^{1}$ School of Radiation Medicine and Protection and Jiangsu Provincial Key Laboratory of Radiation Medicine and Protection; ${ }^{2}$ Collaborative Innovation Center of Radiation Medicine of Jiangsu Higher Education Institutions and School for Radiological and Interdisciplinary Sciences (RAD-X); ${ }^{3}$ School of Public Health, Medical College of Soochow University, Suzhou, Jiangsu 215123; ${ }^{4}$ The Core Laboratory of Suzhou Cancer Center and Department of Radiotherapy of Suzhou Municipal Hospital, Suzhou, Jiangsu 215001, P.R. China
\end{abstract}

Received January 26, 2015; Accepted April 16, 2015

DOI: 10.3892/or.2015.3962

\begin{abstract}
Esophageal squamous cell carcinoma (ESCC), one of the most common gastrointestinal tumors, is known for its high mortality rate. microRNAs (miRNAs) have been reported to play important regulatory roles in cancer metastasis and progression. miR-34a has been demonstrated to be associated with the development of and metastasis in certain types of cancer via various target genes, but its function and targets in ESCC are unknown. The aim of this study was to examine whether the expression of miR-34a was significantly decreased in ESCC tissues, compared with normal esophageal tissues using RT-PCR and western blot analysis. The results showed that miR-34a overexpression increased apoptosis and decreased clonogenic formation, but inhibited invasion and migration in ESCC cells by suppressing MMP-2 and -9 expression. Yin Yang-1 (YY1), a widely distributed transcription factor that belongs to the GLI-Kruppel class of zinc finger proteins, was found to be a direct target of miR-34a in ESCC cell lines. Rescue experiments indicated that the suppressive effect of miR-34a on invasion and migration was mediated by activating YY1 expression. Results of the present study showed that miR-34a is associated with ESCC migration and provides a potential therapeutic and diagnostic target for ESCC.
\end{abstract}

Correspondence to: Professor Jundong Zhou, The Core Laboratory of Suzhou Cancer Center and Department of Radiotherapy of Suzhou Municipal Hospital, 16 Western Baita Road, Suzhou, Jiangsu 215001, P.R. China

E-mail: zhoujundong330@163.com

Professor Jianping Cao, Medical College of Soochow University, 199 Ren'ai Road, Suzhou, Jiangsu 215123, P.R. China

E-mail: jpcao@suda.edu.cn

*Contributed equally

Key words: esophageal squamous cell carcinoma, miR-34a, Ying Yang 1, migration

\section{Introduction}

Esophageal squamous cell carcinoma (ESCC), one of the most prevalent and lethal cancers worldwide, results from the uncontrolled proliferation of cells. Due to its poor prognosis, the 5-year survival rate is $<15 \%$ (1-3). Esophageal carcinogenesis is a multistep process that occurs due to genetic alterations and disturbances of gene expression. In addition, numerous genes have been identified to be essential during carcinogenesis (4). Moreover, in recent years, accumulating evidence has demonstrated that small non-coding RNA microRNAs (miRNAs) and long non-coding RNAs (lncRNAs), as transcript classes in the human genome, may play an important role in tumorigenesis and tumor progression (5-7). However, the underlying mechanisms of ESCC remain poorly understood. Therefore, a better understanding of the detailed mechanisms may be useful in identifying new therapeutic targets and strategies for the treatment of ESCC.

miRNAs, a class of short, single-stranded, non-coding RNA, bind to the complimentary recognition sequences in the 3'-untranslated region (3'UTR) of target mRNA, and are recognized as important post-transcriptional regulators of gene expression (8). miRNA dysregulation can therefore contribute to cell biological processes including migration, invasion, apoptosis and metastasis by affecting relevant transcripts (9-11). It has been reported that miR-34a expression was significantly reduced in non-small cell lung cancer compared with normal tissues (12). miR-34a induces G1 arrest, apoptosis and senescence by repressing the expression of Notch1 in RCC cell lines, and inhibit gastric cancer tumorigenesis by targeting PDGFR and MET through the PI3K/Akt pathway $(13,14)$. miR-34a, which is located at chromosome $1 \mathrm{p} 36$, is a well-known tumor-suppressor miRNA present in various types of human cancer $(15,16)$. However, the detailed role of miR-34a in ESCC remains poorly understood. In the present study, we found that upregulated miR-34a increased apoptosis and decreased clonogenic formation, migration and invasion by directly downregulating Yin Yang-1 (YY1) in TE-1 cells. 


\section{Materials and methods}

Tissue samples. For real-time PCR analysis, 20 normal esophageal and 20 human ESCC tissues were used, as previously reported (17). These tissues were obtained postoperatively between 2010 and 2012 from the Gastrointestinal Center, Jiangyin People's Hospital, Medical School of University of Southeast of China (Jiangyin, China). All the patients provided signed, informed consent for their tissues to be used for scientific research. Ethics approval for the study was obtained from the Jiangyin People's Hospital.

$R N A$ isolation and RT-PCR. Total miRNA was extracted from cultured cells and esophageal tumor and normal esophageal tissues using TRIzol (Invitrogen, Carlsbad, CA, USA). cDNAs were reverse transcribed to cDNA using the RT-PCR kit (GenePharma, Shanghai, China). Quantitative PCR was performed on a CFX96 ${ }^{\mathrm{TM}}$ Real-Time PCR Detection system (ABI 7500; Applied Biosystems, Foster City, CA, USA) using SYBR $^{\circledR}$ Premix Ex Taq ${ }^{\text {TM }}$ II (GenePharma). The following PCR conditions were used: denaturation at $95^{\circ} \mathrm{C}$ for $30 \mathrm{sec}$, followed by 40 cycles of annealing at $95^{\circ} \mathrm{C}$ for $10 \mathrm{sec}$ and extension at $62^{\circ} \mathrm{C}$ for $30 \mathrm{sec}$. The data were normalized against the expression of the U6 snRNA. After amplification, melting curve analysis was performed to ensure the specificity of the products.

Cell culture and transfection. The human ESCC TE-1 cell line was purchased from the Type Culture Collection of the Chinese Academy of Sciences (Shanghai, China). The cell lines were cultured in Dulbecco's modified Eagle's medium (DMEM) supplemented with 10\% fetal bovine serum (FBS) (both from Gibco, Grand Island, NY, USA) and incubated at $37^{\circ} \mathrm{C}$ with $5 \% \mathrm{CO}_{2}$. Cell transfections were performed using Lipofectamine 2000 (Invitrogen-Life Technologies, Carlsbad, CA, USA). Transfected miRNA mimics or inhibitors were purchased from GenePharma. The miRNAs mimic or inhibitor sequences used were : $\mathrm{miR}-34 \mathrm{a}$ mimic, sense,5'-UGGCAGUGU CUUAGCUGGUUGU-3' and antisense, 5'-AACCAGCUAAG ACACUGCAAUU-3'; miR-34a NC, sense, 5'-UUCUCCGAA CGUGUCAGGUTT-3' and antisense, 5'-ACGUGACACGUU CGGAGAATT-3'; miR-34a inhibitor, 5'-ACAACCAGCUAA GACACUGCCA-3'; and miR-34a inhibitor NC, 5'-CAGUAC UUUUGUGUAGUACAA-3'.

Measurement of apoptosis. Apoptosis was measured using propidium iodide (PI)/Annexin V double-staining following the manufacturer's instructions (Keygen Biotech, Nanjing, China). Cells were harvested $24 \mathrm{~h}$ after transfection and apoptotic fractions were measured using flow cytometry (Beckman Coulter, Brea, CA, USA). The Annexin $\mathrm{V}^{+} / \mathrm{PI}^{-}$cells indicated early apoptotis and Annexin $\mathrm{V}^{+} / \mathrm{PI}^{+}$cells late apoptosis. The percentage of the two types of cells was calculated.

Clonogenic formation assay. For the clonogenic formation assay, $1 \times 10^{3}$ cells were seeded in 6-well plates separately. After transfection, the cells were incubated for $\sim 10$ days. The cells were then washed with PBS, fixed and stained with Giemsa. The clone number (cells population $>50$ ) was counted using a microscope.
Western blot analysis. Total protein was extracted from the cells using whole cell lysates (Beyotime, Nantong, China). The protein concentrations of individual samples were assessed using a standard bicinchoninic acid assay (Beyotime). For each sample, $30 \mu \mathrm{g}$ of protein was loaded on a $10 \%$ SDS-PAGE gel (Bio-Rad, Hercules, CA, USA), transferred onto a polyvinylidene difluoride membrane and blocked with 5\% skimmed milk and $0.1 \%$ Tris-buffered saline Tween-20 (TBST) at room temperature for $1.5 \mathrm{~h}$. The membranes were washed in TBST three times and incubated overnight at $4^{\circ} \mathrm{C}$ with rabbit anti-YY1, anti-MMP-2 and anti-MMP-9 antibody (1:1,000 dilution) (all from Santa Cruz Biotechnology, Inc., Santa Cruz, CA, USA) and mouse anti-GAPDH and mouse anti- $\beta$ actin antibody (1:8,000 dilution) (both from Beyotime). The membranes were then washed with TBST and incubated with anti-rabbit IgG horseradish peroxidase-conjugated secondary antibody (Cell Signaling Technology, Boston, MA, USA). The protein expression was evaluated using chemiluminescence and exposure to Kodak film (Kodak, Rochester, NY, USA).

YY1 overexpression plasmids and shRNA. YY1 overexpression was obtained from OriGene Technologies Inc. (Rockville, MD, USA). A shRNA negative vector and two shRNA were constructed. Their targeting sequences are: shRNA-NC, 5'-GTTCTCCGAACGTGTCACGT-3'; shRNA-1,5'-GAACUC ACCUCCUGAUUAU-3'; and shRNA-2, 5'-CAAAGAUGU UCAGGGAUAA-3'.

Luciferase reporter assay. The 3'UTR of YY1 was amplified using the primers: forward, 5'-GCTCTAGAAAAGAAGAGA GAAGACCT-3' and reverse, 5'-GCTCTAGACCATGTAACA GAAAGGGC-3'. The amplified fragmented was inserted at the $X b a \mathrm{I}$ site of pGL3-promoter vector (Promega, Madison, WI, USA). The construct was sequenced for confirmation. For transfection, TE-1 cells were transfected by Lipofectamine 2000 (Invitrogen-Life Technologies). The luciferase reporter containing a fragment of the YY1 3'UTR was co-transfected with pRL-TK (Promega) and synthetic RNAs. Luciferase activity was measured using the Dual-Luciferase Reporter Assay system (Promega). Promoter activities were expressed as the ratio of firefly luciferase to Renilla luciferase activity.

Cell viability assays. Cells were transfected with the indicated RNAs. The transfected cells were seeded in 96-well plates at a density of $1 \times 10^{4}$ cells/well. MTT solution $(20 \mu \mathrm{l}$ of $5 \mathrm{mg} / \mathrm{ml}$ MTT) was added to each well (for a total volume of $100 \mu \mathrm{l}$ ), and the plates were incubated for $4 \mathrm{~h}$ at $37^{\circ} \mathrm{C}$. Following removal of the culture medium, the remaining crystals were dissolved in DMSO, and the absorbance at $490 \mathrm{~nm}$ was measured.

Invasion assays and wound-healing assay. For the invasion assays, $5 \times 10^{4}$ cells were plated in the top chamber containing a Matrigel-coated membrane (24-well insert, 8-mm pore size; BD Biosciences). Twenty-four serum-starved cells were plated in serum-free medium. The medium supplemented with $10 \%$ serum was used as a chemoattractant in the lower chamber. The cells were incubated for $48 \mathrm{~h}$ at $37^{\circ} \mathrm{C}$ in a tissue culture incubator with $5 \% \mathrm{CO}_{2}$. The non-invading cells were removed from the upper sides of the Transwell membrane filter inserts using cotton-tipped swabs. The invaded cells on the lower 
sides of the inserts were stained with Giemsa, and the cells were counted. Sterile ruler and skin marker tip were used to draw a straight width line in a cell-covered plate. The cells were washed with PBS, and cultured with serum-free medium. After $48 \mathrm{~h}$, the width of the line was observed using a microscope. Four random views were taken and quantified under a microscope.

Statistical analysis. The SPSS 17.0 software was used for the statistical analysis. The data are presented as the means \pm SEM. Group comparisons were evaluated by one-way ANOVA to determine statistical significance. Differences were considered significant when $\mathrm{P}<0.05$.

\section{Results}

miR-34a is downregulated in esophageal normal tissues compared with tumor tissues. We first determined the expression of miR-34a in esophageal cancer cells and matched adjacent normal esophageal tissues. miR-34a expression in 20 human ESCC and 20 adjacent normal esophageal tissues was determined by RT-qPCR analysis. Results showed that the levels of miR-34a expression were significantly decreased by 4.46-fold in the tumor tissues compared to the adjacent normal mucosa tissues (Fig. 1). In these samples, miR-34a expression was decreased in 19 ESCC samples, suggesting that the downregulation of miR-34a may be a hallmark of ESCC.

miR-34a inhibits TE-1 cell proliferation, colony formation and apoptosis, as well as migration and invasion. To investigate the effect of miR-34a on TE-1 cell motilities, miRNA-NC, miR-34a mimics, miRNA inhibitor-NC, or miR-34a inhibitor was transfected into TE-1 cells (Fig. 2A and B). miR-34a overexpression reduced the proliferation rate and promoted apoptosis in cultured TE-1 cells (Fig. 2C, G and F). Colony formation of TE-1 cells was significantly decreased following the transfection of miR-34a mimics (Fig. 2D and E). Fortyeight hours after transfection, the wound-healing was determined and the results revealed that the width of line in the miR-34a mimics-transfected group was wider than that of the miRNA-NC-transfected cells. Conversely, the miR-34a inhibitor-transfected group showed the smallest width (Fig. 3A). The results of the invasion assay showed that the invasion cell number in the mimics group had fewer cells than that of the inhibitor group (Fig. 3C and D). Western blotting also demonstrated that miR-34a downregulated MMP-2 and -9 expression (Fig. 3B). These results indicated that miR-34a inhibited migration, invasion and proliferation in ESCC cells.

miR-34a directly targets $Y Y 1$. Bioinformatic tools predicted that human miR-34a may target the 3'UTR of YY1 between position 710 and 726 bp (relative to the YY1 stop codon, Fig. 4A). To further confirm that miR-34a directly targets YY1, we performed luciferase reporter assays to examine whether miR-34a regulates YY1 3'UTR. The 3'UTR of YY1 was cloned and inserted downstream of the pGL3-promoter vector designated as pGL3-YY1 3'UTR. We found that co-transfection of miR-34a with pGL3-YY1 3'UTR caused a significant decrease in luciferase activity compared to the miRNA-NC-

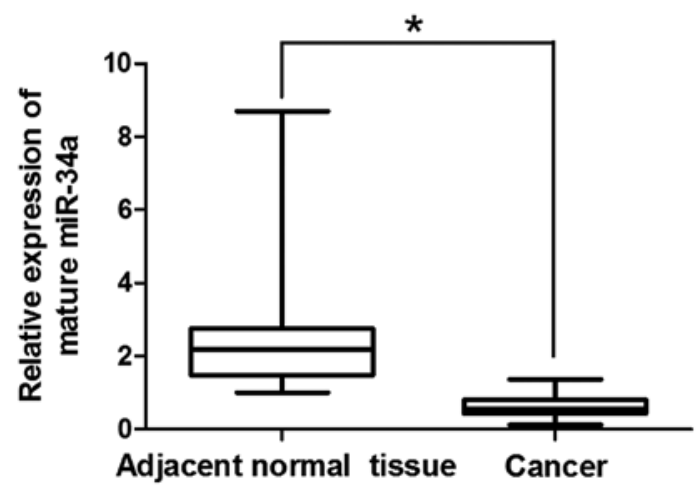

Figure 1. miR-34a is downregulated in esophageal normal tissues compared with tumor tissues. qPCR was used to detect the miR-34a level in adjacent normal esophageal and tumor tissues. miR-34a expression was significantly decreased in esophageal cancer $0{ }^{*} \mathrm{P}<0.05$.

co-transfected cells (Fig. 4B). Conversely, co-transfection of the miR-34a inhibitor increased the luciferase activity, suggesting a suppressing role of miR-34a. Western blotting demonstrated that YY1 expression was significantly decreased in the miR-34a mimics-transfected group, whereas miR-34a inhibitor transfection caused an increase in YY1 expression (Fig. 4C). The result showed that YY1 expression was gradually reduced when the concentration of transfected miR-34a was increased (Fig. 4D).

YY1 promotes TE-1 migation and invasion. We investigated whether YY1 facilitates the metastasis and invasion of ESCC cells. We previously reported that YY1 promotes the invasion of TE-1 cells (18). To confirm this result, TE-1 cells were transfected with pcDNA3.1-YY1 or pcDNA3.1 plus shRNANC or shRNA-YY1. The wound-healing assay showed that YY1 overexpression healed more rapidly than the remaining groups (Fig. 5A), which is consistent with the results of our previous study.

The Transwell-based invasion assay was used to identify the relationship between YY1 and miR-34a. The results showed that co-transfected miR-34a and shRNA-YY1 significantly increased the invasion ability of TE-1 cells (Fig. 5B and C). This result suggested that shRNA targeting YY1 reversed the inhibitory effects of miR-34a on the invasion ability of TE-1 cells. The results from western blotting showed that MMP-2 and -9 expression was correlated with the invasive status of cells. In the present study, MMP-9 expression in the shRNAYY1-transfected group was significantly decreased compared to that of pcDNA3.1-YY1. MMP-9 was markedly increased in co-transfected miR-34a and shRNA-YY1, which is consistent with the result of the invasion assay (Fig. 5D and E).

\section{Discussion}

miRNAs have demonstrated far-reaching effects on the development of cellular biology and cancer $(19,20)$. miR-34a is reported to be significantly dysregulated in various cancer types, such as colon, prostate and pancreatic cancer and glioma (21-23). In the present study, the qPCR examination revealed that miR-34a expression significantly decreased in 13 of the 20 human esophageal tumor tissues compared 
A
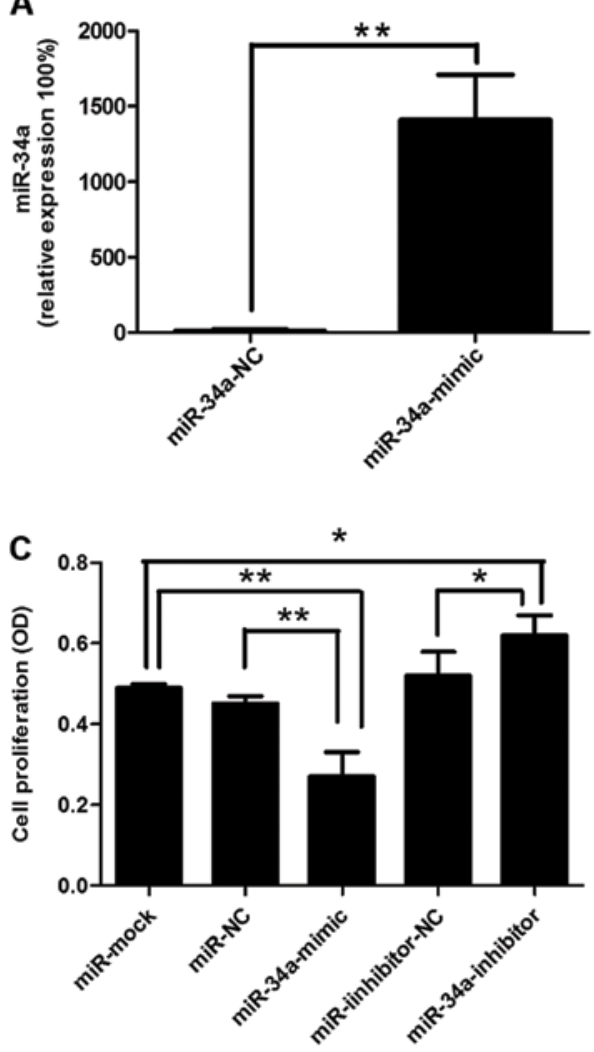

E

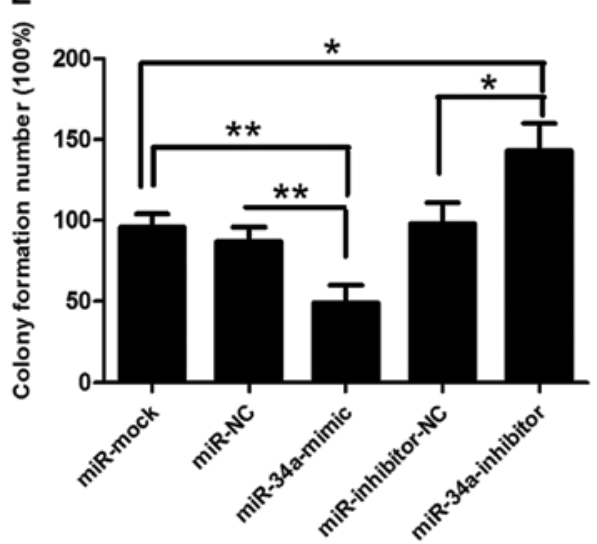

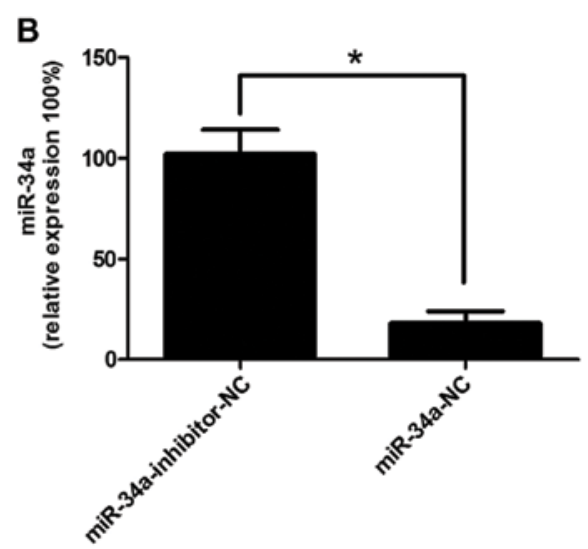

D

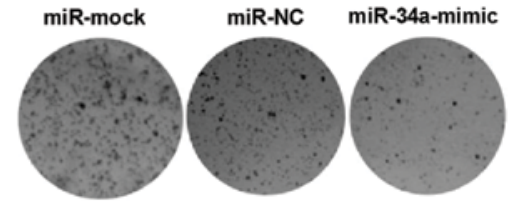

miR-inhibitor-NC miR-34a-inhibitor

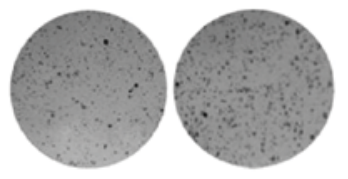

$\mathbf{F}$

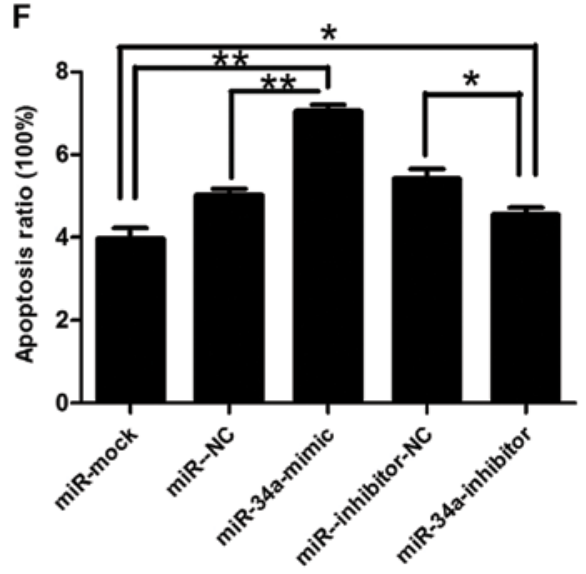

G
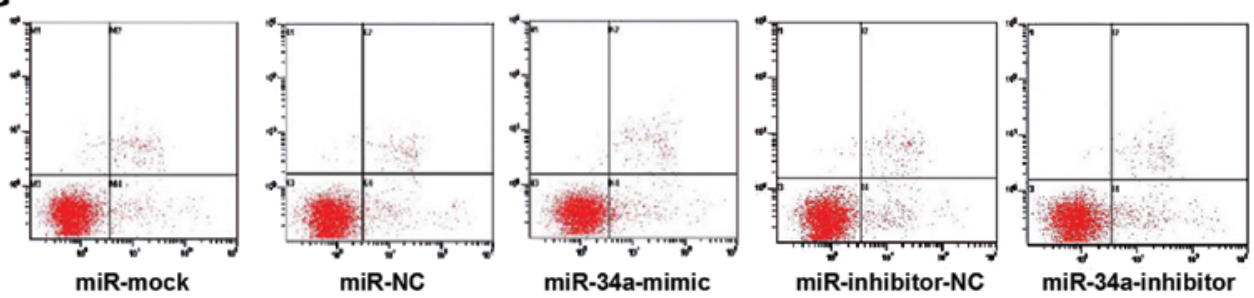

Figure 2. miR-34a inhibits TE-1 cell proliferation, colony formation and apoptosis. (A and B) Relative miR-34a expression in TE-1 cells following the transfection of miR-34a mimics or inhibitors. Relative miR-34a RNA level was determined by qPCR. (C) MTT was used to detect cell proliferation after mock transfection or transfection of miR-34a mimics, miR-NC, miR-inhibitor-NC and miR-34a inhibitor. The proliferation of TE-1 cells was significantly reduced after transfection of miR-34a mimics compared to the other groups. (D and E) Colony formation of TE-1 cells was determined by the clonogenic formation assay. The miR-34a mimics group was significantly decreased compared to the other groups. (F) Apoptosis and necrosis were measured using propidium iodide (PI)/Annexin V double-staining. The apoptotic ratio was increased following the transfection of miR-34a mimics. (G) Representative apoptotic images of indicated groups by flow cytometry.

with the normal tissues, which suggested that dysregulation of miR-34a may be involved in the development of human esophageal cancer. Moreover, studies have reported that
miR-34a can affect the growth of proneural glioma and renal cell carcinoma cells $(13,24)$. In the present study, we found that TE-1 cells grew slowly following transfection with 
A

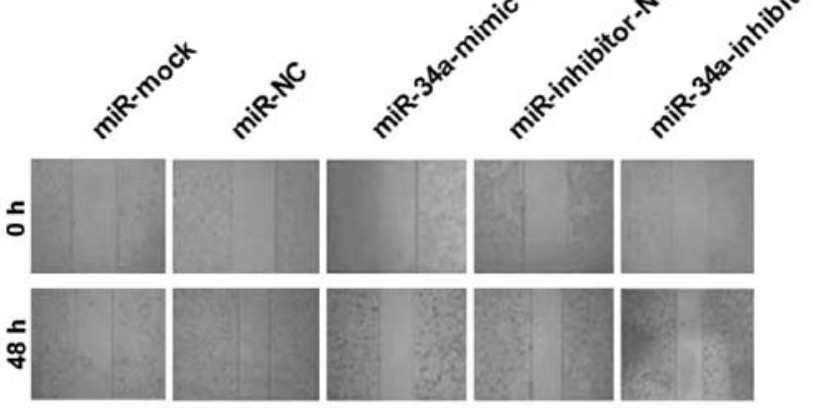

C
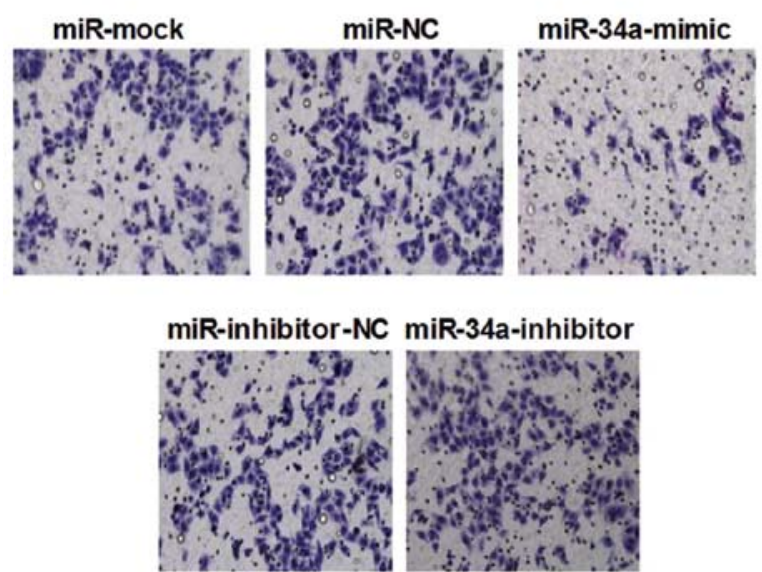

B

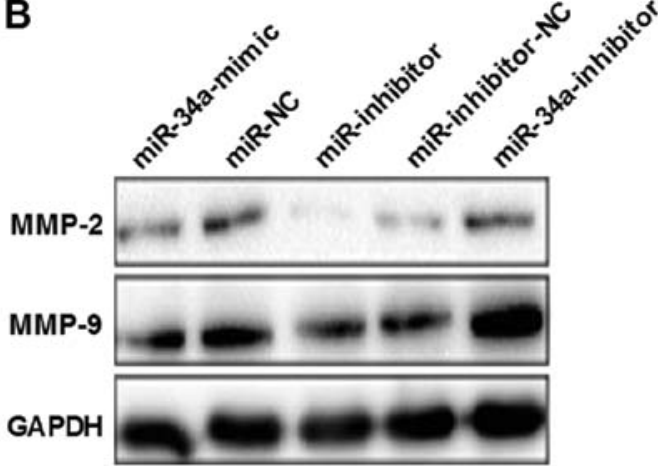

D

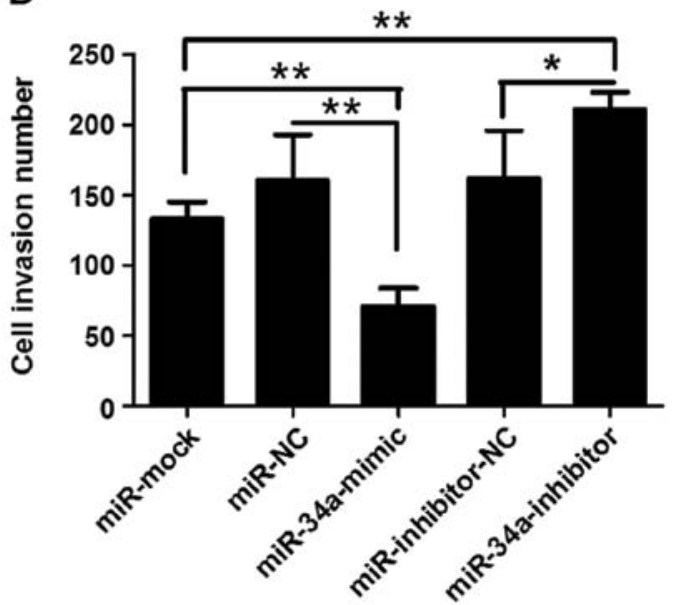

Figure 3. miR-34a inhibits TE-1 cell migration and invasion. (A) Wound-healing assay was observed in transfected cells. Forty-eight hours after transfection, the width in the miRNA mimics NC-transfected group was wider than that of the miR-34a-inhibitor group. (C and D) Invasion assays were performed for $48 \mathrm{~h}$ after transfection with TE-1 cells. Cell numbers show that mimics group has fewer cells than the inhibitor group. (B) Western blotting shows that miR-34a downregulated MMP-2 and -9 expression, ${ }^{*} \mathrm{P}<0.05,{ }^{* *} \mathrm{P}<0.01$

A

\section{YY13'UTR 5'-UUUUUCC-AAAAAAAUACUGCCAG-3' II II I IIIIII| Has-miR-34a 3'-UGUUGGUCGAUUCUGUGACGGU-5'}

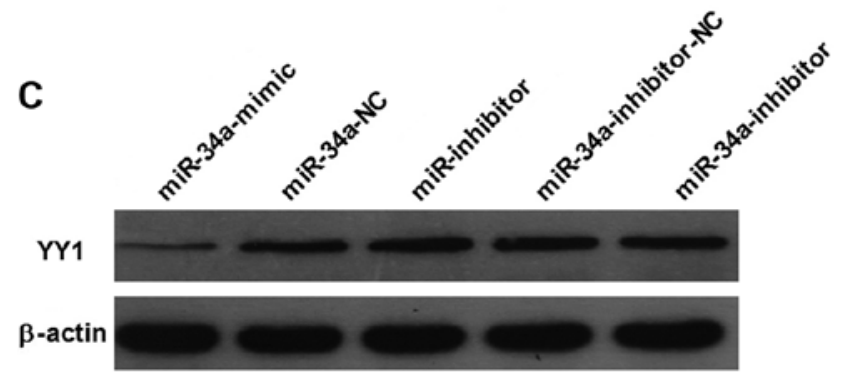

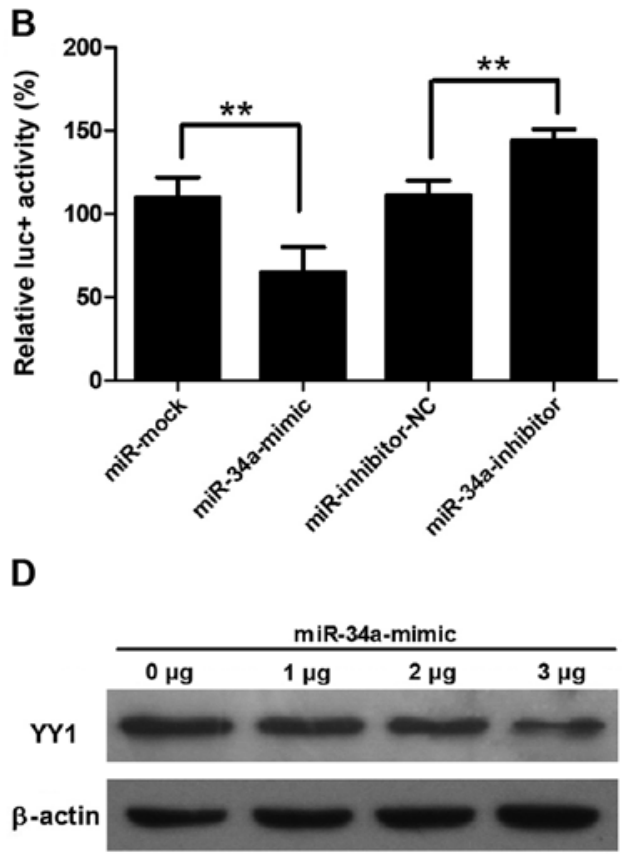

Figure 4. miR-34a directly targets YY1. (A) Bioinformatic tools showed the partial complementarity between YY1 3'UTR and miR-34a. (B) Luciferase activity was measured with the Dual-Luciferase Reporter Assay system. Promoter activities of co-transfectionof miR-34a and pGL3-YY1 3'UTR were significantly decreased in the mimics group compared to the other groups. (C and D) Western blotting showing that YY1 expression was significantly decreased in miR-34a mimics-transfected group and decreased when the concentration of miR-34a was increased. ** $\mathrm{P}<0.01$. 3'UTR, 3'-untranslated region; YY1, Yin Yang-1. 
A

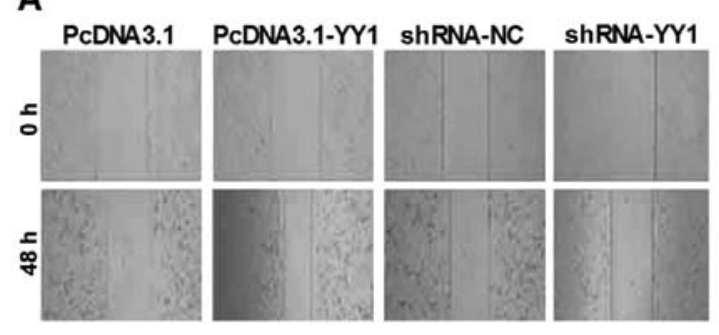

B

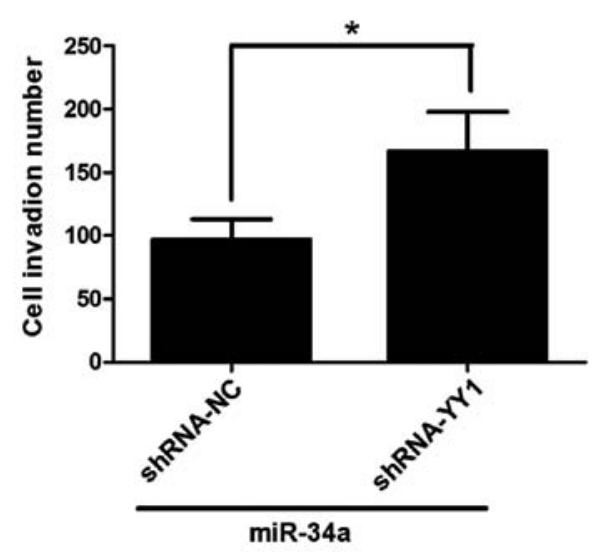

C

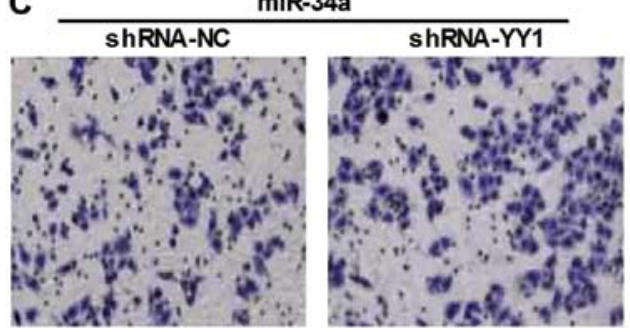

D

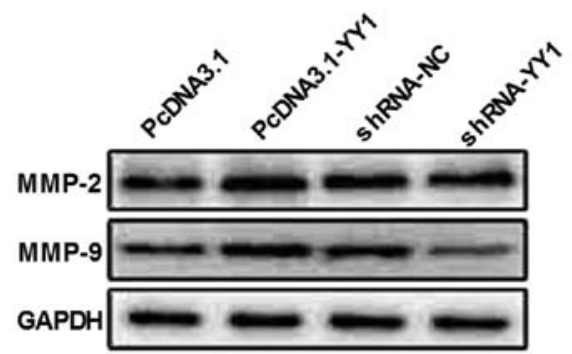

$\mathbf{E}$

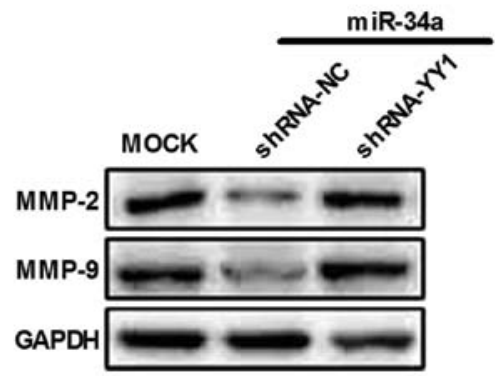

Figure 5. YY1 promotes TE-1 migration and invasion. (A) A wound-healing assay was performed after transfection of indicated vectors. The width of the line in the overexpressed pcDNA3.1-YY1 group was narrower than that of the other groups. (B and C) Invasion of TE-1 cells co-transfected with miR-34a and shRNA-YY1 precursors was assessed in the Matrigel-coated membrane for $48 \mathrm{~h}$. The invasion of the TE-1 cells of the co-transfected miR-34a and shRNA-YY1 groups was significantly increased as compared to that of the miR-34a and shRNA-NC. (D and E) Western blotting was used to detect MMP-2 and -9 expression. MMP-2 expression in the shRNA-YY1 group was significantly increased as compared to shRNA-NC, MMP-9 expression was decreased. "P<0 .05. YY1, Yin Yang-1.

miR-34a mimics. Metastasis is a major cause of death in esophageal cancer patients (25) and the MMP family has been considered to be involved in cancer invasion and metastasis, especially MMP-2 and -9 . Due to their ability to degrade type IV collagen, MMP-2 and -9 have been correlated with the invasive stage of carcinomas (26). It is reported that MMP-2 and -9 were involved in breast cancer initiation and growth in the early stage of tumor genesis (27). In the present study, the cell invasion number and expression of MMP-2 and -9 were significantly decreased when transfected with miR-34a mimics. This result suggested that miR-34a mediated the metastasis of ESCC through MMP-2 and -9.

Moreover, using a bioinformatics approach and a literature review, we found that miR-34a directly regulates numerous target genes. Among them, transcription factor YY1 is likely to be an important target gene of miR-34a. YY1 plays important roles in cell proliferation and differentiation (28). Increased YY1 expression was observed in various types of cancer, such as breast, ovarian, colon, bone, liver, lung, bladder, cervical, prostate and esophageal cancer (29-35). However, the specific function in different cancer types has not been elucidated. In the present study, luciferase activity identified that miR-34a can directly target YY1, and western blotting showed that the expression of YY1 was inhibited by miR-34a. In addition, in the wound-healing and invasion assays, the results suggest that YY1 is an important factor in promoting cell metastasis.

In conclusion, we have demonstrated that the miR-34a/ YY1 axis contributes to esophageal cancer progression by increasing apoptosis and inhibiting clonogenic formation, cell proliferation and invasion. miR-34a negatively regulated YY1 expression in TE-1 cells. These results provide a therapeutic target for esophageal cancer.

\section{Acknowledgements}

This study was supported by the National Natural Science Foundation of China (nos. 81302382, 81472917, 81372433 and 81402626), the Key Programs of Natural Science Foundation of Jiangsu, Educational Committee (no. 11KJA310001), the Priority Academic Program Development of Jiangsu Higher Education Institutions (PAPD) and the Natural Science Fund for Colleges and Universities in Jiangsu Province (no. 12KJB330005).

\section{References}

1. Ferlay J, Shin HR, Bray F, Forman D, Mathers C and Parkin DM: Estimates of worldwide burden of cancer in 2008: GLOBOCAN 2008. Int J Cancer 127: 2893-2917, 2010. 
2. Jemal A, Bray F, Center MM, Ferlay J, Ward E and Forman D: Global cancer statistics. CA Cancer J Clin 61: 69-90, 2011.

3. Lv Y, Zhang J and Qiao L: Quality of life in patients with esophageal cancer receiving definitive chemoradiotherapy or esophagectomy. Mol Clin Oncol 2: 870-874, 2014.

4. Li SQ, Li F, Xiao Y, Wang CM, Tuo L, Hu J, Yang XB, Wang JS, Shi WH, Li X, et al: Comparison of long non coding RNAs microRNAs and messenger RNAs involved in initiation and progression of esophageal squamous cell carcinoma. Mol Med Rep 10: 652-662, 2014.

5. Lu J, Xue L, Jin M and Lyu N: Expression profiling of metastasis-related microRNAs in early esophageal squamous cell carcinoma. Zhonghua Bing Li Xue Za Zhi 43: 313-317, 2014 (In Chinese).

6. Pan F, Yao J, Chen Y, Zhou C, Geng P, Mao H and Fang X: A novel long non-coding RNA FOXCUT and mRNA FOXC1 pair promote progression and predict poor prognosis in esophageal squamous cell carcinoma. Int J Clin Exp Pathol 7: 2838-2849, 2014.

7. Ge H, Lu Y, Chen Y, Zheng X, Wang W and Yu J: ERCC expression and tumor regression predict survival in esophageal squamous cell carcinoma patients receiving combined trimodality therapy. Pathol Res Pract 210: 656-661, 2014.

8. Caporali A and Emanueli C: MicroRNA regulation in angiogenesis. Vascul Pharmacol 55: 79-86, 2011.

9. Banaudha KK and Verma M: The role of microRNAs in the management of liver cancer. Methods Mol Biol 863: 241-251, 2012.

10. Tavazoie SF, Alarcón C, Oskarsson T, Padua D, Wang Q, Bos PD, Gerald WL and Massagué J: Endogenous human microRNAs that suppress breast cancer metastasis. Nature 451: 147-152, 2008.

11. Valastyan S, Reinhardt F, Benaich N, Calogrias D, Szász AM Wang ZC, Brock JE, Richardson AL and Weinberg RA: A pleiotropically acting microRNA, miR-31, inhibits breast cancer metastasis. Cell 137: 1032-1046, 2009.

12. Gallardo E, Navarro A, Viñolas N, Marrades RM, Diaz T, Gel B, Quera A, Bandres E, Garcia-Foncillas J, Ramirez J, et al: miR-34a as a prognostic marker of relapse in surgically resected non-small-cell lung cancer. Carcinogenesis 30: 1903-1909, 2009.

13. Zhang C, Mo R, Yin B, Zhou L, Liu Y and Fan J: Tumor suppressor microRNA-34a inhibits cell proliferation by targeting Notch1 in renal cell carcinoma. Oncol Lett 7: 1689-1694, 2014.

14. Peng Y, Guo JJ, Liu YM and Wu XL: MicroRNA-34a inhibits the growth, invasion and metastasis of gastric cancer by targeting PDGFR and MET expression. Biosci Rep 34: e00112, 2014.

15. Corcoran C, Rani S and O'Driscoll L: miR-34a is an intracellular and exosomal predictive biomarker for response to docetaxel with clinical relevance to prostate cancer progression. Prostate 74 $1320-1334,2014$

16. Daige CL, Wiggins JF, Priddy L, Nelligan-Davis T, Zhao J and Brown D: Systemic delivery of a miR34a mimic as a potential therapeutic for liver cancer. Mol Cancer Ther 13: 2352-2360, 2014.

17. Zhou J, Zhang S, Xie L, Liu P, Xie F, Wu J, Cao J and Ding WQ Overexpression of DNA polymerase iota $(P o l \iota)$ in esophageal squamous cell carcinoma. Cancer Sci 103: 1574-1579, 2012.

18. Luo J, Jiang X, Cao L, Dai K, Zhang S, Ge X, Zhou X and Lu X: Expression of YY1 correlates with progression and metastasis in esophageal squamous cell carcinomas. Onco Targets Ther 7: $1753-1759,2014$.

19. Jones KB, Salah Z, Del Mare S, Galasso M, Gaudio E, Nuovo GJ, Lovat F, LeBlanc K, Palatini J, Randall RL, et al: miRNA signatures associate with pathogenesis and progression of osteosarcoma. Cancer Res 72: 1865-1877, 2012.
20. Maire G, Martin JW, Yoshimoto M, Chilton-MacNeill S, Zielenska $M$ and Squire JA: Analysis of miRNA-gene expression-genomic profiles reveals complex mechanisms of microRNA deregulation in osteosarcoma. Cancer Genet 204: 138-146, 2011.

21. Siemens H, Neumann J, Jackstadt R, Mansmann U, Horst D, Kirchner $\mathrm{T}$ and Hermeking H: Detection of miR-34a promoter methylation in combination with elevated expression of c-Met and $\beta$-catenin predicts distant metastasis of colon cancer. Clin Cancer Res 19: 710-720, 2013.

22. Liu C, Kelnar K, Liu B, Chen X, Calhoun-Davis T, Li H, Patrawala L, Yan H, Jeter C, Honorio S, et al: The microRNA miR-34a inhibits prostate cancer stem cells and metastasis by directly repressing CD44. Nat Med 17: 211-215, 2011.

23. Guessous F, Zhang Y, Kofman A, Catania A, Li Y, Schiff D, Purow B and Abounader R: microRNA-34a is tumor suppressive in brain tumors and glioma stem cells. Cell Cycle 9: 1031-1036, 2010.

24. Silber J, Jacobsen A, Ozawa T, Harinath G, Pedraza A, Sander C, Holland EC and Huse JT: miR-34a repression in proneural malignant gliomas upregulates expression of its target PDGFRA and promotes tumorigenesis. PLoS One 7: e33844, 2012.

25. Jemal A, Siegel R, Ward E, Hao Y, Xu J and Thun MJ: Cancer statistics, 2009. CA Cancer J Clin 59: 225-249, 2009.

26. Stetler-Stevenson WG: Type IV collagenases in tumor invasion and metastasis. Cancer Metastasis Rev 9: 289-303, 1990.

27. Duffy MJ, Maguire TM, Hill A, McDermott E and O'Higgins N: Metalloproteinases: role in breast carcinogenesis, invasion and metastasis. Breast Cancer Res 2: 252-257, 2000.

28. Leeman MF, Curran S and Murray GI: New insights into the roles of matrix metalloproteinases in colorectal cancer development and progression. J Pathol 201: 528-534, 2003.

29. Jeong HM, Lee SH, Yum J, Yeo CY and Lee KY: Smurf2 regulates the degradation of YY1. Biochim Biophys Acta 1843: 2005-2011, 2014

30. Allouche A, Nolens G, Tancredi A, Delacroix L, Mardaga J, Fridman V, Winkler R, Boniver J, Delvenne P and Begon DY: The combined immunodetection of AP-2alpha and YY1 transcription factors is associated with ERBB2 gene overexpression in primary breast tumors. Breast Cancer Res 10: R9, 2008.

31. Zaravinos A and Spandidos DA: Yin yang 1 expression in human tumors. Cell Cycle 9: 512-522, 2010.

32. Kashyap V and Bonavida B: Role of YY1 in the pathogenesis of prostate cancer and correlation with bioinformatic data sets of gene expression. Genes Cancer 5: 71-83, 2014.

33. Huerta-Yepez S,Liu H, Baritaki S, DelLourdes Cebrera-Muñoz M, Rivera-Pazos C, Maldonado-Valenzuela A, Valencia-Hipolito A, Vega MI, Chen H, Berenson JR, et al: Overexpression of Yin Yang 1 in bone marrow-derived human multiple myeloma and its clinical significance. Int J Oncol 45: 1184-1192, 2014.

34. Notarbartolo M, Giannitrapani L, Vivona N, Poma P, Labbozzetta M, Florena AM, Porcasi R, Muggeo VM, Sandonato L, Cervello M, et al: Frequent alteration of the Yin Yang 1/Raf-1 kinase inhibitory protein ratio in hepatocellular carcinoma. OMICS 15: 267-272, 2011.

35. Luo J, Zhou X, Ge X, Liu P, Cao J, Lu X, Ling Y and Zhang S: Upregulation of Ying Yang 1 (YY1) suppresses esophageal squamous cell carcinoma development through heme oxygenase-1. Cancer Sci 104: 1544-1551, 2013. 